\title{
Flujos de remesas y COVID-19 en América Latina. Reflexiones al inicio de la pandemia
}

\section{Remittances Flows and COVID-19 in Latin America. Reflections at the Start of the Pandemic}

\author{
Adriana Cardozo-Silva \\ Luis Díaz-Pavez \\ University of Goettingen \\ Inmaculada Martínez-Zarzoso \\ University of Goettingen \\ Universitat Jaume I
}

\section{Resumen}

Las remesas que los inmigrantes envían a su país de origen contribuyen a alcanzar los $\mathrm{Ob}$ jetivos de Desarrollo Sostenible (ODS), permitiendo que muchas familias salgan de la pobreza. Dichos envíos han disminuido en la primera mitad de 2020 debido a la crisis de la COVID-19. Centrándonos en un grupo de países de América Latina, en este artículo hacemos un análisis exploratorio sobre el efecto que la COVID-19 podría tener sobre las remesas a través de la contracción en el PIB y el empleo de los países que las envían. Así mismo, relacionamos dicho impacto con la rigurosidad de las medidas tomadas en cada país y comparamos la evolución observada hasta ahora con lo ocurrido tras la crisis financiera global de 2008.

Palabras clave: remesas, Latinoamérica, COVID-19, confinamiento.

Clasificación JEL: F10, O10.

\section{Abstract}

The money immigrants send back to their countries -remittances-could contribute to achieve the Sustainable Development Goals (SDG) allowing many families to escape poverty. These remittances have decreased in the first half of 2020 due to the COVID-19 crisis. Focusing on a group of Latin American countries, we present an exploratory analysis of the effect that COVID-19 could have on remittances through the contraction in GDP and employment in the sending countries. Likewise, we relate this impact to the stringency of the lockdown measures taken in each country and compare the evolution observed so far with the aftermaths of the global financial crisis of 2008.

Keywords: remittances, Latin America, COVID-19, lockdown.

JEL Classification: F10, O10. 


\section{Introducción}

La rápida expansión y virulencia de la COVID-19, declarada por la OMC como pandemia el 11 de marzo de 2020, sorprendió a las economías de todos los continentes, empezando por Asia, pasando por Europa y desembocando en las Américas. Ya en enero, el virus comienza a traspasar fronteras en Asia y en febrero se extiende a Europa, donde el incremento exponencial de los contagios lleva a tomar medidas de confinamiento, con la clausura de las actividades no esenciales. En España el confinamiento se extiende desde el 15 de marzo hasta mediados de mayo. La gravedad de la crisis con caída de la demanda y shock de oferta obliga a los Gobiernos a tomar medidas de política fiscal expansiva y al Banco Central Europeo (BCE) políticas de expansión monetaria, además de brindar ayudas a las familias y a las empresas.

En América Latina, el primer caso de COVID-19 se identificó en Brasil a finales de febrero y, desde entonces, se ha extendido por toda la región, donde algunos países han impuesto confinamientos muy estrictos y prolongados, como Argentina, Chile y Perú, mientras que otros han sido más laxos, como Brasil y México. La región lo tiene más difícil que Europa porque muchos países partían de una situación macroeconómica inestable y porque, en general, poseen un reducido margen fiscal además de mayores dificultades para acceder a financiación externa.

Una fuente de financiación importante para las familias con pocos recursos son las remesas de los emigrantes. Dichas familias se han visto doblemente afectadas por la pandemia. Por un lado, se ha incrementado la vulnerabilidad de los emigrantes, cuyos trabajos se han visto interrumpidos por el confinamiento, con la consecuente reducción en el envío de remesas a sus familiares. Por otro lado, los ingresos y el empleo han caído abruptamente debido a los confinamientos en los países de origen.

La literatura sobre la dinámica de las remesas resalta su resiliencia ante crisis económicas y su efecto anticíclico en respuesta a las contracciones del Producto Interior Bruto (PIB) o a desastres naturales en los países de origen de los migrantes (Ahmed y Martínez-Zarzoso, 2016). Aunque en el corto plazo se observe una recuperación rápida en el volumen de los envíos tras los confinamientos en la primera mitad del 2020, es muy posible que la caída global del empleo causada por la COVID-19, tanto en los países receptores de remesas como en los países desde los cuales se envían, tenga un impacto aún más profundo que el observado en la crisis financiera global de 2008, limitando el efecto amortiguador de las remesas. Sobre todo, considerando la -ya confirmada- entrada en una segunda ola de contagios masivos en octubre de 2020 que va a desencadenar nuevos confinamientos.

La principal contribución de este trabajo consiste en presentar una revisión de la bibliografía y de los indicadores disponibles para avanzar en el estudio del impacto que la COVID-19 ha tenido sobre el envío de remesas a Latinoamérica. Para ello, se han seleccionado 7 países en la región ${ }^{1}$ con datos mensuales disponibles de los flujos de remesas enviados en el primer semestre de 2020 y cuya cuantía y evolución se

\footnotetext{
${ }^{1}$ Bolivia, Colombia, El Salvador, Guatemala, México, Paraguay y República Dominicana.
} 
ponen en perspectiva en relación con la severidad de la crisis y las medidas tomadas en los países dónde se originan los envíos. Así mismo, se evalúa el impacto de la COVID-19 en la economía en comparación con lo sucedido tras la crisis financiera global del 2008. El estudio es de carácter exploratorio y tiene como objetivo abrir nuevas líneas de investigación en cuanto al efecto de la pandemia sobre las remesas, en una de las regiones del mundo con mayor desigualdad de oportunidades y en la cual dichos envíos juegan un papel central para mitigar la pobreza.

Para abordar el tema, en el segundo apartado se realiza una breve revisión de los estudios académicos sobre los determinantes y el impacto macroeconómico de las remesas, con especial referencia a los que se centran en países latinoamericanos. En el tercer apartado se presenta un análisis descriptivo y gráfico de la evolución de las remesas durante la crisis, las medidas de confinamiento adoptadas y la evolución de los principales indicadores macroeconómicos en los países analizados. En el cuarto apartado, se realiza una comparación con la crisis financiera global del 2008, con el fin de reflexionar sobre la dinámica de las remesas en tiempos de crisis. Finalmente, el último apartado concluye y propone algunas medidas de política económica, sugiriendo, además, posibles líneas de investigación.

\section{Remesas: determinantes y efectos macroeconómicos en América Latina}

Los envíos de remesas hacia Latinoamérica y el Caribe han aumentado significativamente en las últimas dos décadas de la mano de un crecimiento sostenido en los flujos migratorios. Es así como las remesas se han convertido para algunos países en la principal fuente de divisas, jugando un papel relevante en el crecimiento económico y en la reducción de la pobreza. En esta sección se revisa la bibliografía que estudia los factores más importantes que determinan los flujos de remesas para, a continuación, revisar los estudios que se centran en analizar empíricamente los efectos macroeconómicos de las remesas, prestando particular atención a la región que nos ocupa.

Los trabajos que analizan los determinantes de las remesas suelen incluir factores altruistas e individualistas para explicar el volumen de los flujos (Rapoport y Docquier, 2006; Lueth y Ruiz-Arranz, 2008, entre otros). Así mismo, la literatura señala que las remesas tienden a aumentar como respuesta a catástrofes naturales en los países de origen, por lo cual juegan un papel amortiguador y ayudan a los hogares a mantener el consumo en el medio plazo (Ahmed y Martínez-Zarzoso, 2016; Mohapatra et al., 2012). Sin embargo, no solo la actividad económica y las necesidades en los países de origen de los migrantes afectan el volumen de las remesas. Ahmed et al. (2020) realizan el primer estudio que incluye los costes de transacción entre las variables consideradas para una muestra global de países en el periodo 2011-2017 y encuentran que las remesas enviadas aumentan más que proporcionalmente cuando se reduce el coste del envío. En el caso concreto de Latinoamérica, según Vacaflores (2018), la actividad económica de los países de destino de los migrantes también tiene un efecto positivo y estadísticamente significativo sobre el monto de las remesas 
que recibe la región. Además, las remesas parecen estar directamente relacionadas con las tasas de interés de los países receptores, siendo este un factor individualista que influye en la cuantía de los envíos (Mccracken et al., 2017; Vacaflores, 2018).

Los efectos macroeconómicos de las remesas han sido ampliamente analizados en la literatura de economía del desarrollo, como muestran dos recientes sinopsis de la misma (Rapoport y Docquier, 2006; y Cazachevici et al., 2020). Rapoport y Docquier (2006) presentan un resumen de los aspectos más importantes en torno a las remesas y sus efectos. Sus principales conclusiones son que la migración y las remesas asociadas a esta tienden a tener un efecto positivo en el desempeño económico a largo plazo en los países de origen, mientras que, en el corto plazo, resulta mucho más complejo estimar los efectos macro, ya que dependen del tipo de régimen cambiario, del grado de movilidad en el mercado de capitales y de la flexibilidad del mercado de trabajo. En su reciente trabajo, Cazachevici et al. (2020), presentan un meta-análisis de 95 artículos publicados sobre el efecto de las remesas en el crecimiento económico concluyendo que es positivo, aunque pequeño en magnitud y variable por regiones. Mientras que el efecto es claro en Asia, no lo es tanto en África y Latinoamérica.

Chami et al. (2003) es uno de los primeros estudios globales disponibles que estudia el impacto de las remesas para 113 países. Aunque los autores encuentran un efecto negativo sobre el PIB per cápita, el mismo pasa a ser positivo cuando se considera la calidad de las instituciones. Trabajos más recientes, que tienen en cuenta endogeneidad de las remesas en los modelos estimados, muestran resultados positivos incluso sin condicionar los efectos a la calidad de las instituciones (Catrinescu, 2009; Cruz-Zuniga, 2011).

Para el caso latinoamericano, Ramírez y Sharma (2008) muestran que las remesas tienen un impacto positivo y significativo en el crecimiento de la renta per cápita de los países analizados, siendo el efecto mayor en magnitud para los países con un sistema financiero más desarrollado. Estudios anteriores obtienen resultados similares sobre el crecimiento económico e indican que las remesas contribuyen también a reducir la pobreza y la desigualdad. Acosta et al. (2008) cuantifican el efecto de las remesas para 10 países latinoamericanos y concluyen que estas no solo han reducido la pobreza y la desigualdad, sino además, han contribuido al crecimiento económico, aunque de manera diferenciada dada la heterogeneidad de las economías analizadas. Estos autores encuentran que un aumento promedio de un punto porcentual en las remesas como proporción del PIB, induce una reducción de la pobreza de aproximadamente $0,3 \%$. Estudios específicos por país de origen confirman que las remesas reducen la pobreza, pero también señalan que estas pueden llevar a una menor participación laboral en los hogares receptores, como se puede observar en Haití (Jadotte y Morilla, 2016) y en los países del Triángulo del Norte: El Salvador, Guatemala y Honduras (Sousa y García, 2018).

El impacto de las remesas sobre otros indicadores socioeconómicos ha sido también objeto de estudio en la literatura económica. En cuanto al impacto sobre la educación, la mayoría de estudios encuentran un impacto positivo sobre la tasa de 
escolaridad ante un aumento en el ingreso de los hogares, aunque también hay evidencia de mayores tasas de deserción debido a la ausencia de padres provocada por la migración y a la necesidad de compensar ciertas tareas en el hogar (Adams, 2011; Rapoport y Docquier, 2006; Amuedo-Dorantes y Pozo, 2010; y Bucheli et al., 2018). Respecto al impacto de las remesas sobre indicadores de salud, Acosta et al. (2008) analizan econométricamente el caso de Guatemala y Nicaragua (dado que las encuestas de hogares de dichos países presentan información de indicadores de salud), encontrando efectos positivos y significativos de las remesas tanto en el peso como en la estatura de los niños.

\section{Remesas en América Latina, COVID-19 y crisis económica}

El mayor volumen de remesas en la región lo recibe México, con aproximadamente 38,5 millones de dólares en el 2019, seguido por Guatemala con 10,6 y República Dominicana con 7,2. Sin embargo, como proporción del PIB, las remesas son de vital importancia para economías más pequeñas, siendo Haití el país para el cual las remesas alcanzaron la mayor proporción del PIB (37\%) en el 2019, seguido por Honduras $(22 \%)$ y El Salvador $(21 \%)^{2}$.

Los principales países de procedencia de las remesas varían dentro de la región, siendo Estados Unidos el más importante para los países centroamericanos, mientras que en la región Andina la procedencia está más diversificada y se reparte entre Estados Unidos, Europa y otros países latinoamericanos. Para los países del Cono Sur, sin embargo, Europa cobra también importancia. Así mismo, la tendencia creciente de la migración sur-sur ha tenido también su impacto en el envío de remesas. En Latinoamérica y Caribe (LAC), la crisis económica, política y social de Venezuela explica la migración de aproximadamente 5 millones de venezolanos hacia otros países de la región en la última década, principalmente hacia Colombia, revirtiendo por completo el carácter de país de destino para migrantes colombianos observado hacia finales del siglo xx e invirtiendo la dirección de los flujos de remesas (Caruso et al., 2020). Así mismo, importantes flujos migratorios de Bolivia, Paraguay y Perú hacia los países del Cono Sur han generado corredores de remesas que no existían dos décadas atrás -o cuyos montos eran bastante bajos.

Los flujos financieros internacionales en general, y en particular, los flujos de remesas se han visto afectados por las medidas con las que los Gobiernos han respondido a la pandemia para proteger la salud pública. Sectores como la construcción, el turismo y los pequeños comercios, que tradicionalmente emplean migrantes, han cerrado o restringido su actividad por un periodo prolongado, impactando negativamente en los ingresos de los migrantes o llevando a la pérdida del empleo. A continuación, se presentan una serie de hechos estilizados que reflejan lo acontecido

\footnotetext{
${ }^{2}$ Cálculos del Banco Mundial basados en datos de balanzas de pagos del FMI, de los bancos centrales, y de las agencias nacionales de estadística. https://www.knomad.org/data/remittances
} 
durante el inicio de la pandemia, en cifras: la evolución de las remesas, la intensidad del confinamiento y los impactos de corto plazo sobre los principales determinantes macroeconómicos de las remesas.

\subsection{Evolución de las remesas en el primer semestre de 2020}

La selección de países para el análisis descriptivo se basa en la disponibilidad de datos mensuales de remesas hasta junio de 2020. Aunque no ha sido posible incluir a toda la región, las economías que se presentan en este estudio son muy relevantes en cuanto al tema de análisis. En Centroamérica, contamos con datos para El Salvador, Guatemala y República Dominicana, economías que tienen en común estar altamente dolarizadas, ser relativamente pequeñas y para las cuales las remesas son una proporción alta del PIB (mayor al $10 \%$ en los tres casos). Por otra parte, se incluye México y Colombia, países con volúmenes de remesas altos, pero que, como proporción del PIB, son inferiores al $3 \%$. Por último, la muestra incluye Bolivia y Paraguay que recibieron 1.339 y 668 millones de dólares en remesas en el 2019, procedentes, en gran medida, de los países del Cono Sur.

Como se puede observar en el diagrama de Sankey (Gráfico 1), México, El Salvador y Guatemala reciben remesas predominantemente de Estados Unidos, mientras que para República Dominicana el origen de las remesas está algo más diversificado, siendo España la segunda fuente más importante. En Colombia, la diversificación es

\section{GRÁFICO 1}

FLUJOS BILATERALES DE REMESAS DURANTE EL PRIMER SEMESTRE DE 2020

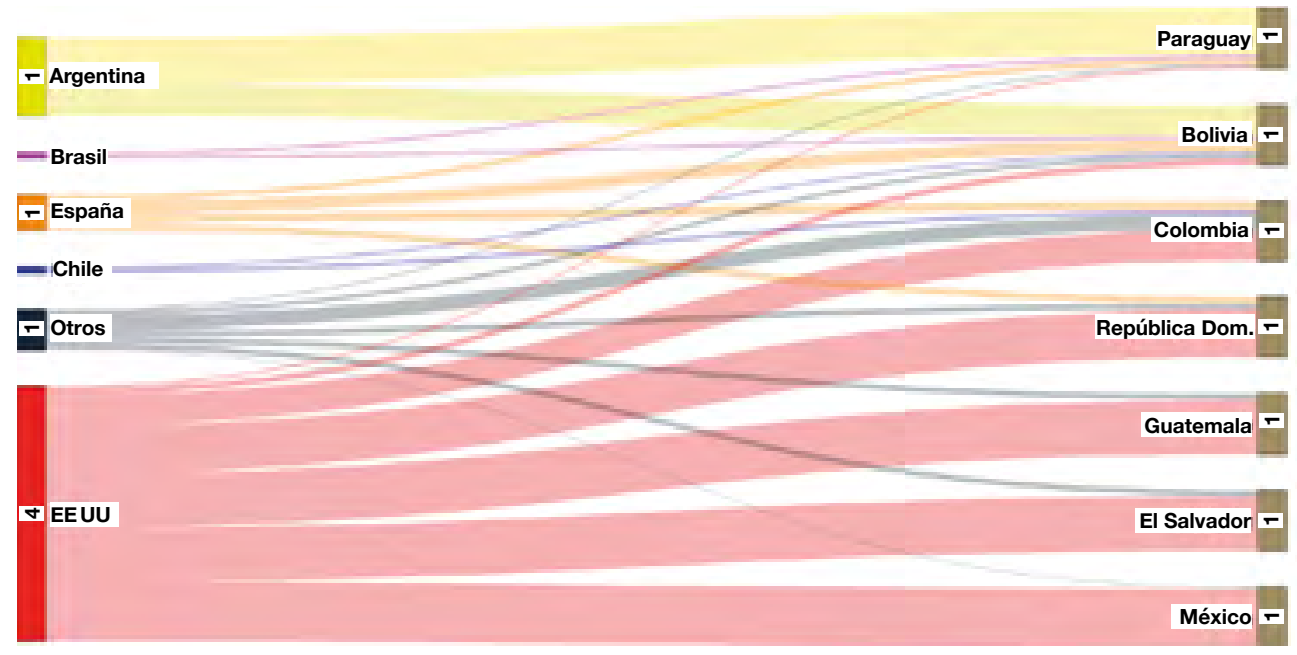

FUENTE: Elaboración propia en base a datos oficiales de remesas de los bancos centrales y a la matriz de remesas bilaterales del Banco Mundial. 
mayor, ya que recibe una importante proporción de remesas de países diferentes a Estados Unidos y España. Por otro lado, los envíos de remesas a Paraguay y Bolivia provienen principalmente de Argentina ${ }^{3}$.

En cuanto a la evolución de las remesas durante la crisis de la COVID-19, podemos observar en el Gráfico 2 que estas sufrieron una caída pronunciada en los primeros meses de la crisis, especialmente en abril, cuando muchos de los países de donde provienen los envíos estaban en pleno confinamiento, entre ellos España y Estados Unidos. Cabe destacar que las mayores caídas interanuales las tuvieron Paraguay y Bolivia, con más del $60 \%$ en abril de 2020, seguidos por El Salvador y Colombia donde se situaron en torno al $40 \%$. Para México, sin embargo, se observa una menor contracción, apenas cercana al $3 \%$, precedida de un aumento del $30 \%$ en marzo, explicado, en parte, por la evolución del tipo de cambio peso-dólar y potencialmente por las ayudas directas del Gobierno estadounidense a los migrantes con permiso de residencia.

El Gráfico 2 muestra que las caídas interanuales fueron menos pronunciadas después de abril, e incluso mostraron ligeros incrementos interanuales. Si se considera el total de remesas recibidas en los primeros seis meses del año, la variación interanual sigue siendo negativa para El Salvador $(-8 \%)$ y Colombia $(-5,3 \%)$. La evolución de las remesas mensuales para el periodo (agosto 2019-2020) confirma, por tanto, la singularidad de la caída interanual producto de la crisis de la COVID-19.

De acuerdo con estimaciones del Banco Mundial se espera una disminución global del 20\% en los flujos de remesas en 2020 (Ratha et al., 2020). En el caso concreto de Latinoamérica, se predice una caída del 19,3\% para todo el año. Las tendencias del tercer trimestre de 2020 podrían hacer pensar en una posible recuperación rápida en las remesas. Sin embargo, la llegada de una segunda ola del virus a principios de octubre de 2020, con una prontitud y virulencia mayor a la esperada lo pone en duda. De ahí surge la diferencia principal entre esta crisis, en términos de un mayor grado de incertidumbre, y la pasada que no dependía de factores exógenos. De hecho, tras la crisis económica de 2008, aunque las remesas en la región cayeron un 12,3\% en el bienio posterior, a ello prosiguió un crecimiento sostenido hasta el 2019. En particular, en dicho periodo las remesas hacia Latinoamérica y el Caribe aumentaron considerablemente, llegando a ser la fuente fundamental de divisas para algunos países centroamericanos y del Caribe, y sobrepasando en muchos casos los flujos de ayuda al desarrollo y de inversión extranjera directa. En esta comparativa profundizaremos en el apartado 4.

${ }^{3}$ La migración paraguaya de mano de obra laboral hacia Argentina, existente desde mediados del siglo $\mathrm{xx}$, se ha intensificado considerablemente en las últimas dos décadas. La tasa de crecimiento anual de migrantes paraguayos en Argentina alcanzó 61,5\% en el 2010, correspondiendo a 44,2\% de los inmigrantes del país (OIM, 2013). 


\section{GRÁFICO 2}

\section{VARIACIÓN PORCENTUAL INTERANUAL DE REMESAS RECIBIDAS}

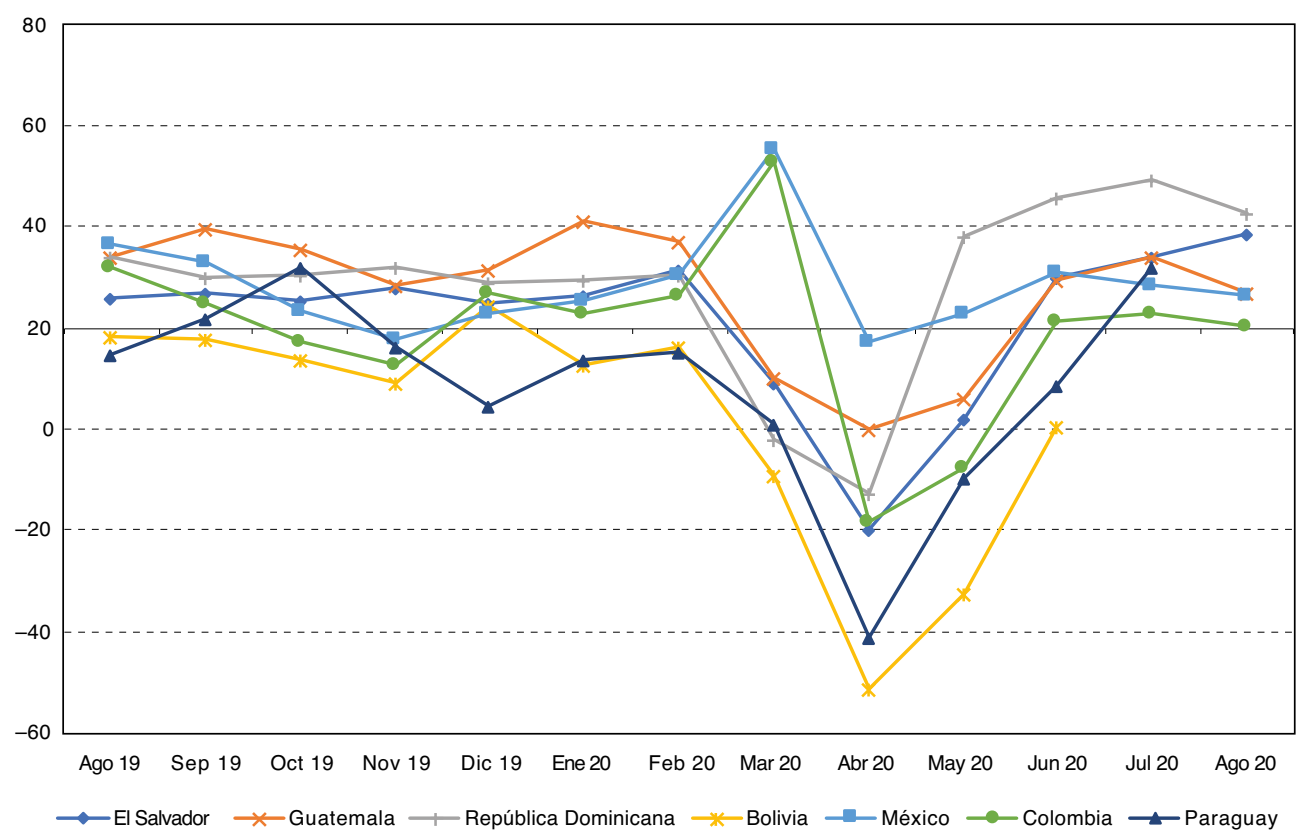

FUENTE: Elaboración propia en base a datos oficiales de remesas de los bancos centrales.

\subsection{Intensidad del confinamiento e incidencia de la COVID-19}

La incidencia de la COVID-19 junto con las subsecuentes medidas de confinamiento han sido particularmente severas tanto en los países de América Latina como en los países donde trabajan gran parte de sus migrantes, generando así un doble impacto en las economías latinoamericanas, principalmente en los sectores más vulnerables de la población. En primer lugar, el impacto es directo y se ve reflejado en la contracción de la actividad económica y del consumo; el aumento de la tasa de desempleo; y la ralentización del sector informal. En segundo lugar, un impacto añadido viene de la pérdida del mecanismo de amortiguación económica que representan las remesas, las cuales operan como un mecanismo eficiente de suavización del consumo.

En cuanto a la incidencia de la COVID-19 por 100.000 habitantes, en el Gráfico 3 se puede observar que esta ha sido mayor en los países que envían remesas en relación con los que las reciben. Así, por ejemplo, en el segundo trimestre del año Chile presentó una tasa de incidencia cercana a los 1.500 casos por 100000 habitantes, seguido por Estados Unidos con una tasa cercana a 750, Brasil con una tasa cercana a 700 y España con una tasa cercana a 350. Mientras tanto, los países que reciben remesas presentaron tasas comparativamente menores, que fluctúan entre 50 (Paraguay) y 300 (Ecuador). 


\section{GRÁFICO 3 \\ INCIDENCIA DE LA COVID-19 POR 100.000 HABITANTES}

Países que envían remesas

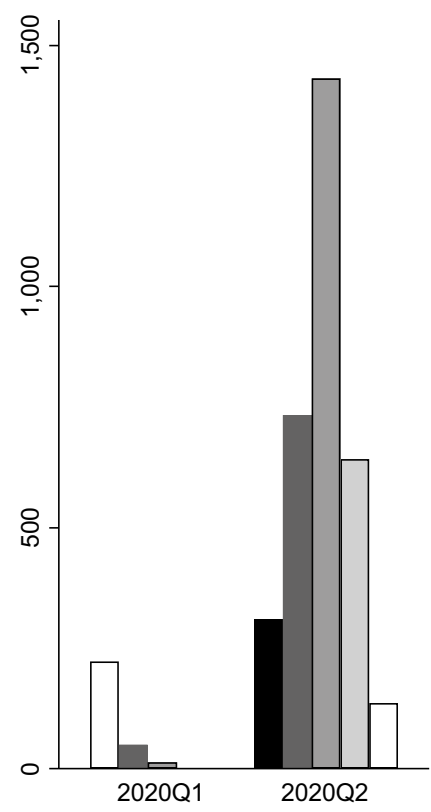

Países que reciben remesas

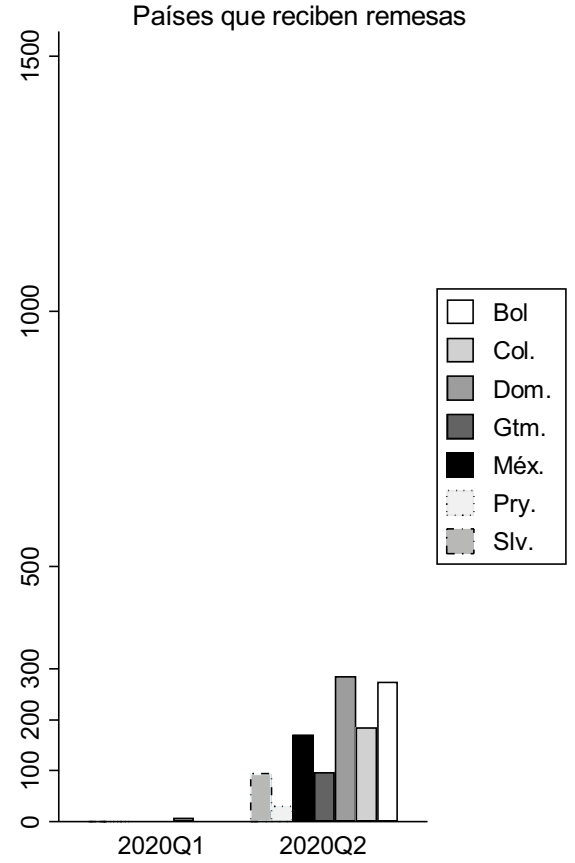

FUENTE: Elaboración propia en base a datos de OWID (Our World in Data) y de la división de estadísticas de la ONU.

La rigurosidad de la respuesta de los Gobiernos ante la COVID-19 puede ser resumida en el «Índice de Rigurosidad» desarrollado por la Universidad de Oxford, el cual incorpora nueve indicadores, tales como cierre de escuelas cierre de lugares de trabajo y restricciones de viaje, entre otros. En el Gráfico 4 se puede observar que tanto los países remitentes como los receptores presentan elevados niveles del índice, que, en general, alcanza su máximo en abril. Es interesante observar que los países receptores analizados presentan índices mayores de rigurosidad que los países remitentes, lo que se explicaría por los mayores resguardos necesarios para evitar el colapso de los ya frágiles sistemas de salud. En cuanto a los países seleccionados que envían remesas, el Índice de Rigurosidad de Argentina es el más alto en el periodo observado. Esto, sumado a la ya alta incidencia de la COVID-19 en Paraguay y Bolivia, que reciben una porción importante de remesas desde Argentina, podría explicar parcialmente la drástica caída de las mismas en dichos países durante los meses de confinamiento. Asimismo, el cierre temporal de agencias receptoras de remesas en dichos meses, siendo estas actividades consideradas como no esenciales, podría también haber contribuido a la caída. 


\section{GRÁFICO 4 \\ ÍNDICE DE RIGUROSIDAD DE POLÍTICAS DE CONFINAMIENTO \\ EN 2020}
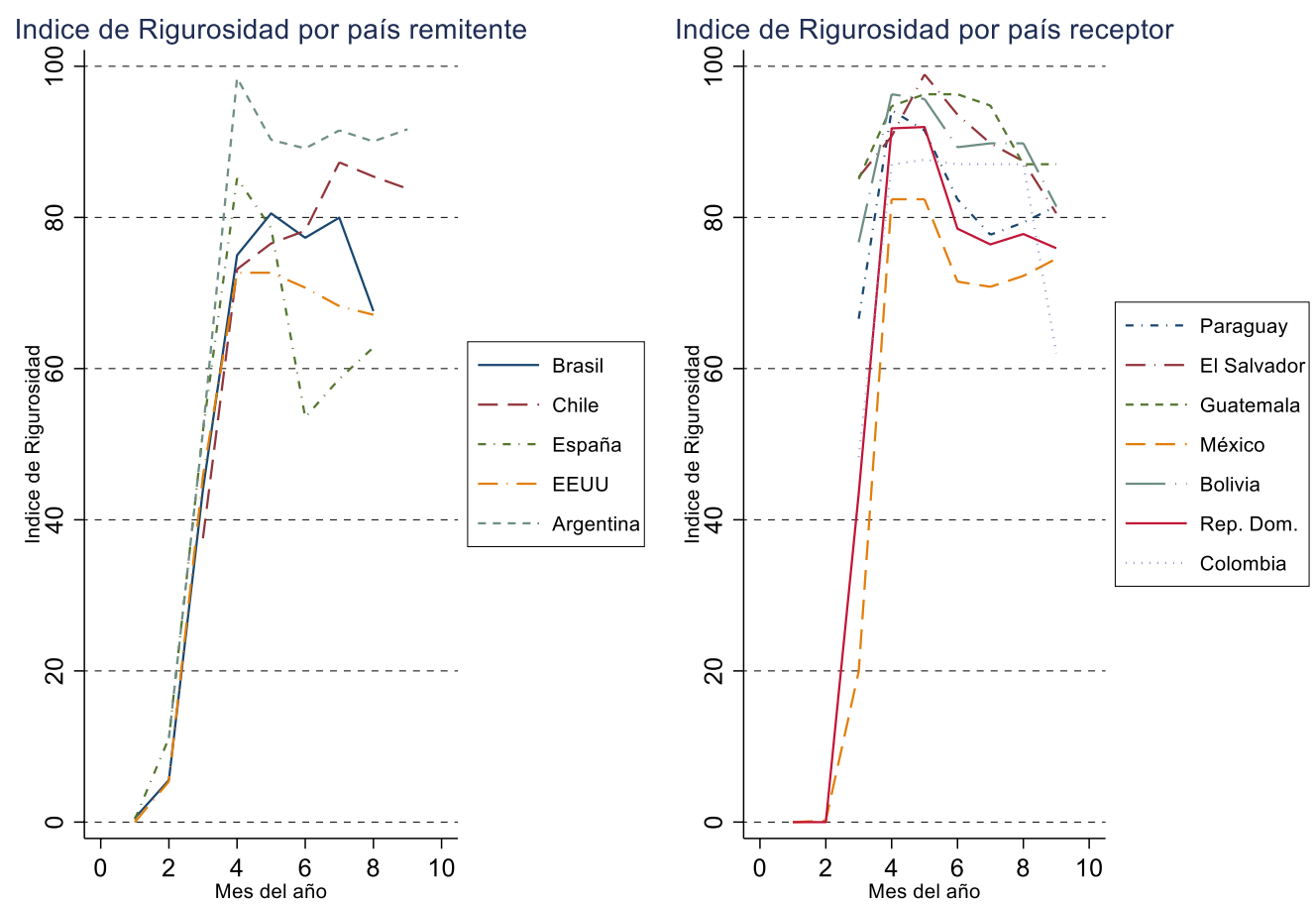

FUENTE: Elaboración propia en base a datos de Oxford COVID-19 Government Response Tracker (OxCGRT) de la Universidad de Oxford.

Por el contrario, tanto México como Estados Unidos han tenido según este índice políticas menos restrictivas, lo cual, sumado a una fuerte depreciación del tipo de cambio en México, podría explicar que sus remesas cayeran proporcionalmente menos que en los demás países del análisis. Respecto a dicho efecto, este operaría vía un aumento del valor en moneda local de los dólares enviados, lo que incrementaría el beneficio esperado de enviar remesas.

\subsection{Efectos macroeconómicos y su relación con las remesas}

En cuanto a los indicadores macroeconómicos en los países que envían remesas, y de acuerdo a los estudios empíricos revisados, los que más afectan la cuantía de los envíos son el PIB y la tasa de desempleo. En el Cuadro 1, se observa cómo la contracción que se inició en el primer trimestre del 2020 se profundizó en el segundo debido a los efectos del confinamiento durante los meses de marzo y abril. Estados Unidos experimentó la mayor caída en el PIB real $(31,7 \%)$, seguido por España 


\section{CUADRO 1}

VARIACIÓN PORCENTUAL INTERANUAL DEL PIB REAL DE LOS PRINCIPALES PAÍSES REMITENTES Y RECEPTORES

\begin{tabular}{|lc|c|c|c|c|c|}
\cline { 2 - 8 } \multicolumn{1}{c|}{} & \multicolumn{4}{c|}{ 2019 } & \multicolumn{2}{c|}{$\mathbf{2 0 2 0}$} \\
\hline Países remitentes & Trim 1 & Trim 2 & Trim 3 & Trim 4 & Trim 1 & Trim 2 \\
\hline Chile & 2,2 & 2 & 1,9 & 1,8 & $-4,1$ & $-21,5$ \\
\hline Brasil & 0,11 & 0,94 & 0,77 & $-4,15$ & 2,98 & $-13,22$ \\
\hline Estados Unidos & 0,59 & 1,08 & 1,2 & 1,67 & $-0,25$ & $-11,44$ \\
\hline Argentina* & 2,9 & 1,5 & 2,6 & 2,4 & -5 & $-31,7$ \\
\hline \multicolumn{1}{|c|}{ Países receptores } & -6 & 0,3 & $-1,8$ & -1 & $-5,4$ & $-19,6$ \\
\hline Bolivia & Trim 1 & Trim 2 & Trim 3 & Trim 4 & Trim 1 & Trim 2 \\
\hline República Dominicana & 3,09 & 2,64 & 2,23 & 1,06 & 0,56 & $-21,68$ \\
\hline El Salvador & 5,73 & 3,75 & 4,92 & 5,80 & 0,02 & $-16,89$ \\
\hline Guatemala & 2,19 & 1,63 & 2,90 & 2,80 & 1,05 & $-19,34$ \\
\hline Paraguay & 3,60 & 3,84 & 4,05 & 3,89 & 0,92 & $-9,64$ \\
\hline México & $-2,66$ & $-3,34$ & 2,60 & 3,49 & 4,36 & $-6,45$ \\
\hline Colombia & 0,08 & $-0,02$ & $-0,46$ & $-0,79$ & $-2,13$ & $-18,68$ \\
\hline
\end{tabular}

NOTA: * Para Argentina las cifras están calculadas respecto al promedio trimestral del índice mensual de actividad económica.

FUENTE: Elaboración propia en base a datos de los bancos centrales y de los institutos nacionales de estadísticas.

$(21,5 \%)$ y Argentina $(19,6 \%)$. Para los países receptores esta tendencia fue similar, siendo Bolivia, El Salvador y México los más afectados, con caídas en el PIB real de $21,7 \%, 19,3 \%$ y $18,7 \%$ respectivamente.

Una de las consecuencias inmediatas de la reducción en la actividad económica producto del confinamiento fue el aumento del desempleo, cuya evolución para los principales países remitentes se presenta en el Gráfico 5. Aquí, se observa un marcado aumento del desempleo en todos los países analizados, principalmente en Estados Unidos, que pasa de 3,8\% en el primer trimestre del año hasta una tasa de $13 \%$ en el segundo trimestre. En dicho país, según el Economic Policy Institute ${ }^{4}$, la tasa de desempleo fue particularmente elevada entre la población latina, llegando a niveles de $16.7 \%$ en hombres y $20,2 \%$ en mujeres, lo que podría ser un factor explicativo de la fuerte reducción en remesas hacía países como El Salvador, Guatemala, y República Dominicana.

\footnotetext{
4 https://www.epi.org/
} 


\section{GRÁFICO 5 \\ TASA DE DESEMPLEO EN PAÍSES REMITENTES DURANTE LA CRISIS COVID-19}

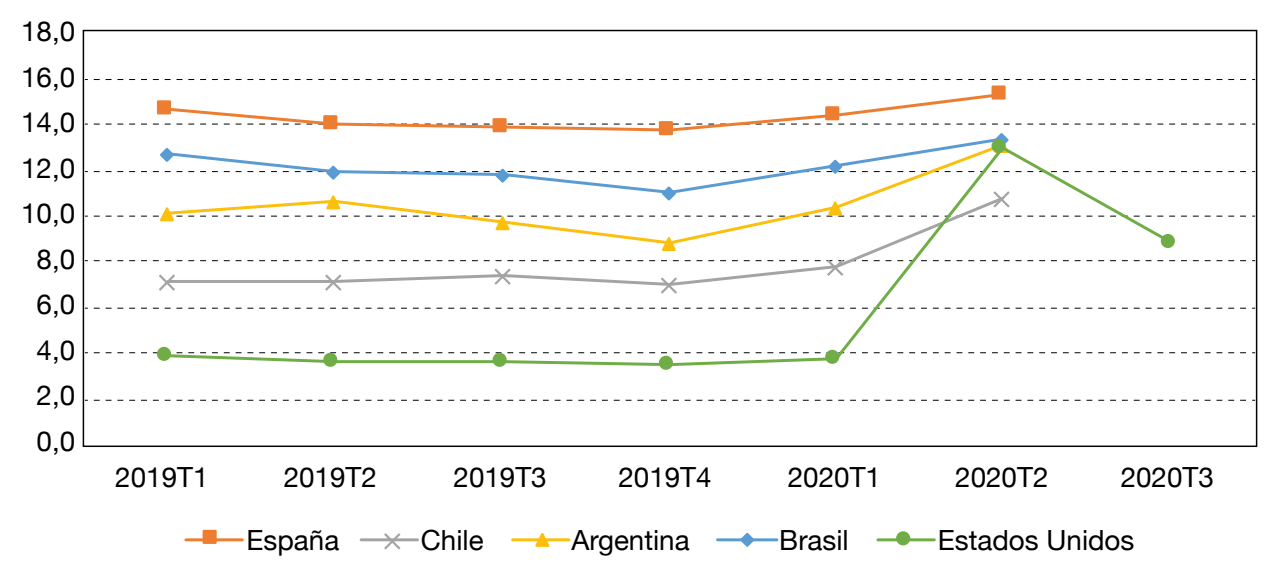

FUENTE: Elaboración propia en base a datos oficiales de los institutos nacionales de estadísticas.

Un factor importante que compensa de manera transitoria la caída en las remesas generada por la reducción del empleo en los países remitentes es la depreciación de las monedas de los países receptores, tal como ha ocurrido en los países productores de petróleo, cuyas economías se han visto además afectadas por la reducción en los precios internacionales del crudo. En particular, para aprovechar los diferenciales cambiarios favorables al dólar y al euro, los migrantes pueden utilizar ahorros o créditos en dichas monedas con el fin de mantener o incluso incrementar los flujos de remesas hacia sus países de origen, tal como se observó durante la crisis financiera global del 2008, lo que contribuyó a que las remesas tuvieran un efecto anticíclico (Buch y Kuckulenz, 2010). Este es el caso de países como Colombia y México, en los cuales el tipo de cambio depende de una combinación compleja de factores internos y externos, y donde la depreciación de la moneda puede llegar a amortiguar una menor capacidad de enviar remesas de los migrantes.

El Gráfico 6 ilustra la evolución del tipo de cambio real para cada país en relación con una canasta de monedas de sus socios comerciales entre agosto de 2019 y julio de $2020^{5}$. Tanto México como Colombia muestran una fuerte depreciación de su moneda en los primeros 3-4 meses de 2020 seguida de una ligera apreciación, mientras que para Paraguay y Bolivia se observa la tendencia opuesta con una apreciación del tipo de cambio real seguida por una depreciación, más pronunciada en el primer país. Guatemala y El Salvador en cambio reflejan una mayor estabilidad en su tipo de cambio, debido en parte al alto grado de dolarización de sus economías, además de

5 Un aumento del índice denota una apreciación de la moneda local en relación con la canasta de monedas de los socios comerciales. 


\section{GRÁFICO 6}

VARIACIÓN PORCENTUAL INTERANUAL DEL TIPO DE CAMBIO REAL

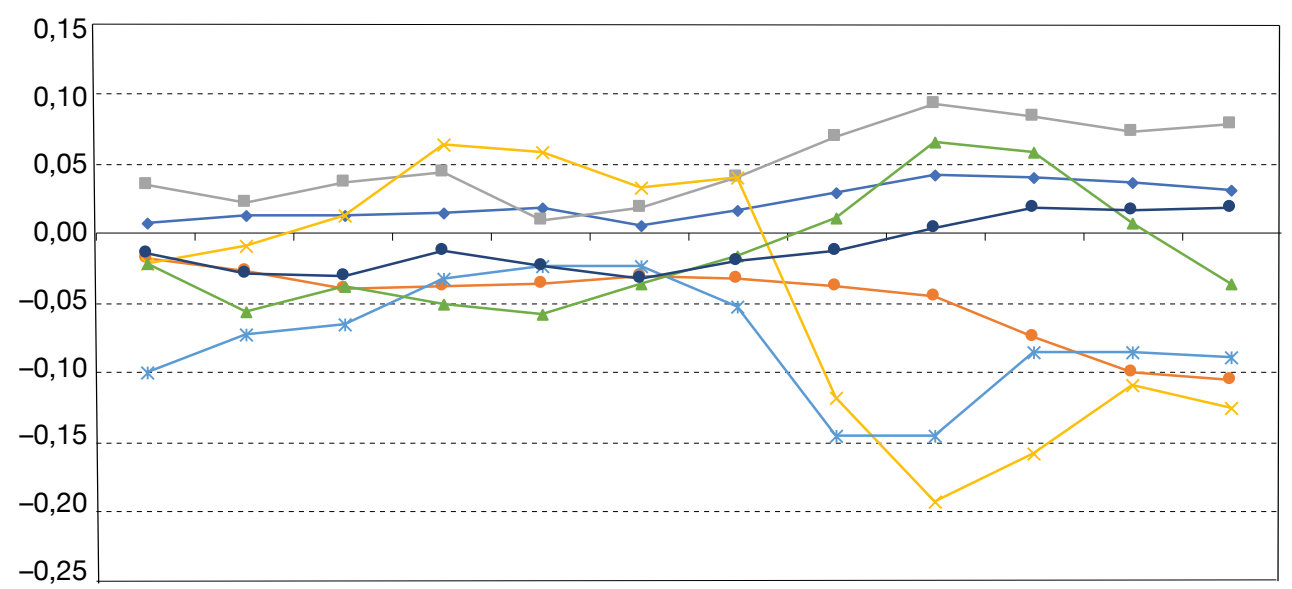

Ago 19 Sep 19 Oct 19 Nov 19 Dic 19 Ene 20 Feb 20 Mar 20 Abr 20 May 20 Jun 20 Jul 20

$\rightarrow$ Guatem. $\rightarrow$ Rep. Dom. - Bol. $\rightarrow$ Méx. $\rightarrow$ Colom. $\rightarrow$ Parag. $\rightarrow$ El Sal.

FUENTE: Elaboración propia en base a datos de Bruegel (2020).

tener a Estados Unidos como su principal socio comercial y fuente de divisas. Es así como, aunque no hay un patrón único en la evolución del tipo de cambio, es posible que las remesas se estén ajustando a las fluctuaciones de este, y que los trabajadores migrantes aprovechen la depreciación de la moneda local, para mantener el flujo de remesas hacía sus países de origen, al menos temporalmente.

\section{Similitudes con la crisis financiera global de 2008}

A diferencia de la crisis generada por la COVID-19, la crisis financiera global (CFG) que se desencadenó en agosto de 2007 afectó inicialmente solo a los países industrializados a través del colapso del sector hipotecario estadounidense y debido a problemas estructurales del sector financiero, generando una falta de liquidez a nivel mundial y provocando a su vez una disminución del comercio internacional y de los flujos de capital. La CFG se intensificó en septiembre de 2008 con el colapso de Lehman Brothers, afectando profundamente los flujos de capital y generando un efecto de contagio a nivel global. Dicho efecto desencadenó fugas masivas de capitales con una recomposición de activos de riesgo hacia activos seguros. La respuesta de la política monetaria se enfocó en activar la demanda en un entorno de contracción del crédito y de tasas de interés históricamente bajas. En comparación con la crisis generada por la COVID-19, la CFG no fue ni global ni enteramente exógena y no ocasionó una contracción simultanea de la oferta y la demanda. Además, en lo que va del año, el impacto económico de la COVID-19 ha sido mucho más profundo que el observado en la CFG de 2008. 
El Gráfico 7 ilustra este escenario para los principales países remitentes de la región, en donde se hace visible la gran diferencia en términos de contracción del PIB. Dos fenómenos se hacen evidentes: el primero es que la caída de la actividad ha sido mucho más repentina durante la crisis COVID, y el segundo es que dicha caída ha sido mucho más severa. De esta forma, mientras que en el peor periodo de la CFG las contracciones en el PIB real variaron en un rango de $-2,2 \%$ para Brasil y $-11,3 \%$ para Argentina en términos interanuales, en la crisis COVID las contracciones variaron entre $-11,4 \%$ para Brasil y $-31,7 \%$ para Estados Unidos durante el segundo trimestre del 2020.

En cuanto al empleo, la COVID-19 ha afectado en mayor medida al sector de servicios, en particular, a la restauración, al turismo y al entretenimiento, principales fuentes de empleo para los migrantes, mientras que la CFG afectó más fuertemente al sector inmobiliario y financiero. Por esta razón, se espera que el impacto de la COVID-19 sobre el desempleo de los migrantes y sobre las remesas sea más duradero y profundo que en la CFG. Además, y tal como se mencionó en el apartado anterior, aunque en la última década la migración tendió a diversificarse, Estados Unidos, España y Argentina siguen siendo los principales países de destino de los migrantes latinoamericanos y donde se originan el $83 \%$ de las remesas ${ }^{6}$.

\section{GRÁFICO 7 \\ VARIACIÓN PORCENTUAL INTERANUAL DEL PIB REAL: \\ CFG VERSUS CRISIS COVID-19}

(En \%)
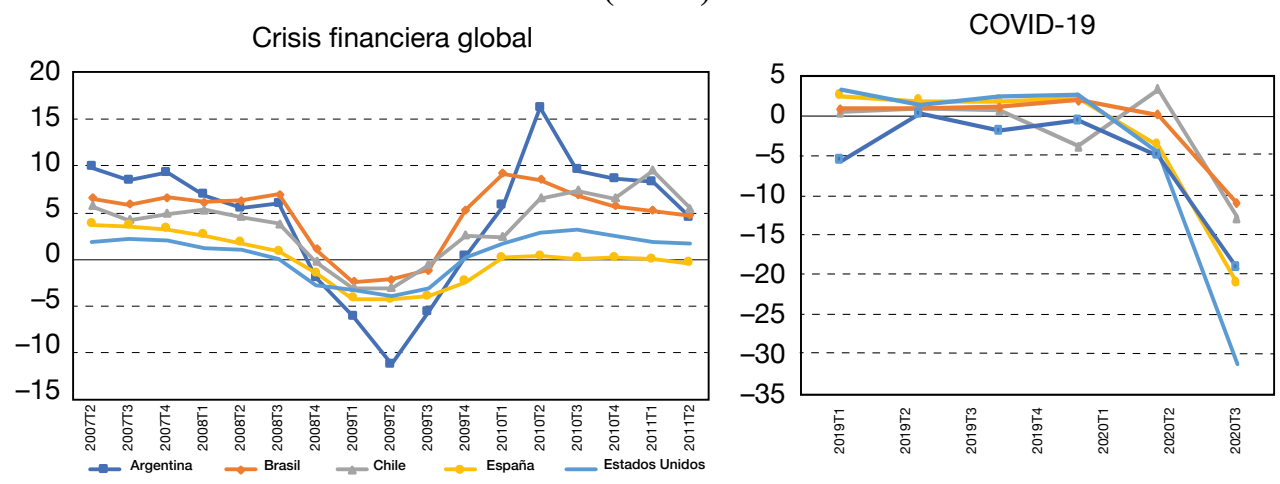

FUENTE: Elaboración propia en base a datos oficiales de los bancos centrales para el periodo 2019T1-2020T3 y datos de las Estadísticas Financieras Internacionales del FMI para el periodo 2007T2-2011T2.

\footnotetext{
${ }^{6}$ Cálculo realizado en base a datos de los bancos centrales y la matriz bilateral de remesas del Banco Mundial.
} 
El aumento del desempleo durante la COVID-19 en EEUU es también superior al experimentado durante la CFG, sobre todo, para la población de origen hispano y latino. Tal como se observa en el Gráfico 8 , mientras el desempleo aumentó de manera sostenida durante el 2008 y 2009, empezando a ceder apenas en el 2011; durante la crisis de la COVID-19 el aumento en el desempleo fue abrupto e inesperado. En concreto, se pasa de tasas alrededor del $4 \%$ en febrero de 2020 en Estados Unidos a más del $18 \%$ tan solo dos meses después con una tasa de desempleo de $13 \%$ para el segundo trimestre, aunque con una rápida recuperación en la segunda mitad del año. Una situación similar, aunque menos abrupta se observa para el resto de los países remitentes.

Durante la CFG, la desaceleración del PIB en las principales economías de destino de los migrantes se transformó gradualmente en un menor flujo de remesas hacia LAC, no solo debido a mayores tasas de desempleo sino a una caída temporal en los flujos migratorios (Fix et al., 2009). Tal como se observa en el Gráfico 9, aunque las remesas enviadas a la región crecieron en promedio 6\% en 2007 respecto al año anterior y se mantuvieron estables en la primera mitad de 2008 , fue en la segunda mitad de 2008 cuando comenzaron a experimentar fuertes contracciones que alcanzaron un promedio de $-11,14 \%$ para toda LAC en el 2009 , coincidiendo con el descenso

\section{GRÁFICO 8 \\ EVOLUCIÓN DE LA TASA DE DESEMPLEO ENTRE 2007 Y 2011 \\ (En \%)}

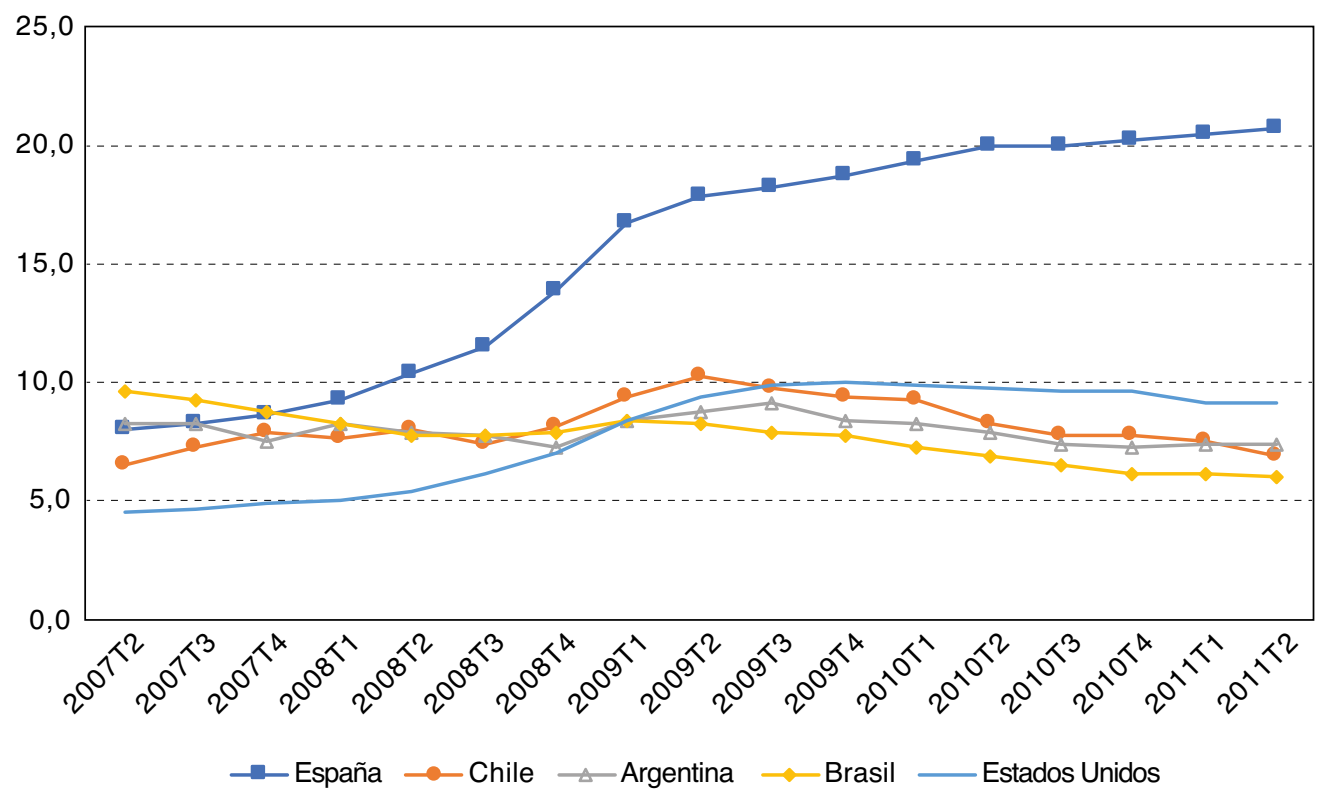

FUENTE: Elaboración propia en base a datos oficiales de los institutos nacionales de estadística de los países considerados. 


\section{GRÁFICO 9 \\ VARIACIÓN PORCENTUAL INTERANUAL DE LAS REMESAS RECIBIDAS ENTRE 2007 Y 2011 \\ (En \%)}

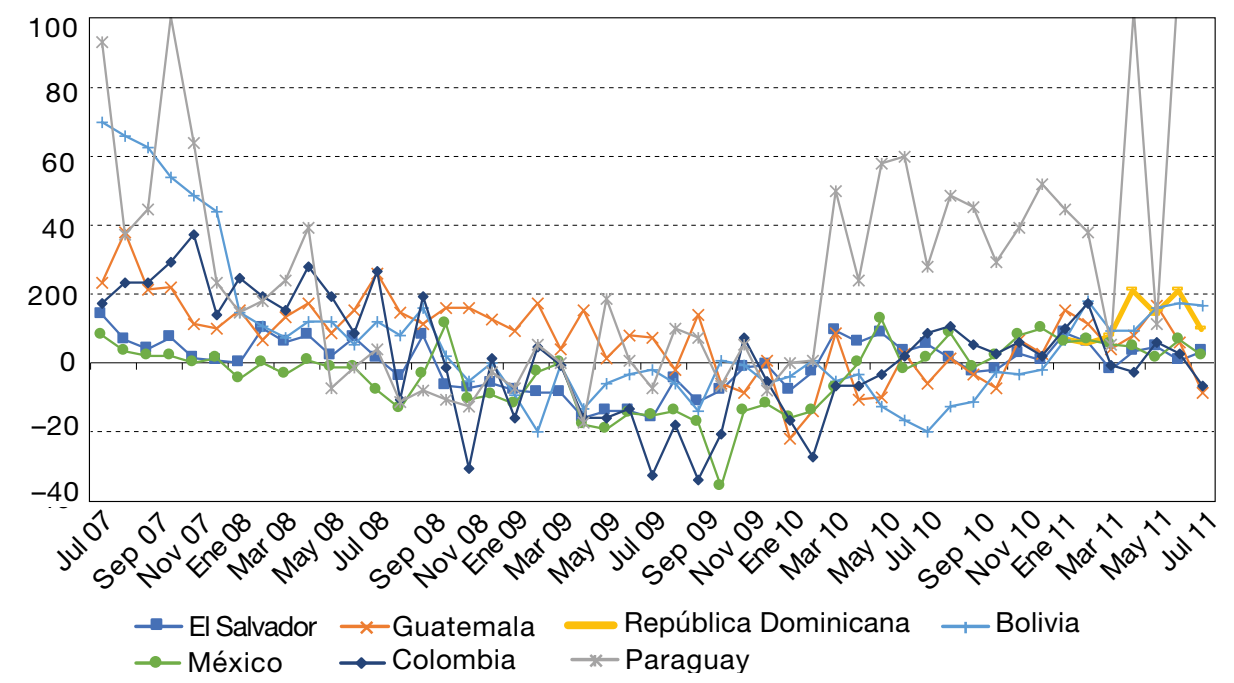

NOTA: Para República Dominicana se reportan valores desde enero del 2011, debido a disponibilidad de datos. FUENTE: Elaboración propia en base a datos oficiales de remesas de los bancos centrales.

de los precios de exportación de las materias primas. Posteriormente, las remesas experimentaron un crecimiento dinámico y sostenido impulsado por una mayor migración, causada en gran parte por el deterioro de las condiciones socioeconómicas en los países de LAC. Tan solo en Ecuador, más de medio millón de personas emigraron, principalmente hacia España y EEUU (Bertoli y Marchetta, 2014).

Tal como se mencionó en el segundo apartado, los trabajos revisados señalan que la resiliencia de las remesas ante las crisis económicas es mayor a la de los flujos privados de capital (Sirkeci et al., 2012). La evidencia indica que, en tiempos de crisis, los inmigrantes establecidos en los países de destino utilizan sus ahorros para mantener las remesas y no retornan a sus países de origen, incluso ante pérdidas del empleo. Sin embargo, Lim y Mahbub (2015) señalan que el aumento de las remesas poscrisis se debe no tanto a un mayor volumen de remesas de los migrantes que ya residían en los países de destino, sino, en gran parte, a una mayor migración desde los países afectados. En comparación con la CFG, la migración internacional se suspendió abruptamente durante el 2020 debido a las restricciones internacionales de movilidad. Aunque puede preverse que la intención de migrar aumente en los próximos años debido al deterioro en las condiciones económicas en los países de LAC, es también plausible que se dé un escenario tanto con políticas migratorias más estrictas como con menores oportunidades de empleo en los países de destino como respuesta a las propias crisis domésticas. 
En conclusión, se encuentran algunas similitudes con la CFG, en donde tanto las economías avanzadas como las desarrolladas sufrieron una importante caída del PIB. Sin embargo, la magnitud de la caída es mayor en la actual crisis COVID-19 y la recuperación para los países en desarrollo puede llegar a ser más lenta, aumentando el diferencial de ingreso entre países ricos y pobres y motivando así una nueva ola migratoria.

\section{Conclusiones y agenda para futuras investigaciones}

En un mundo globalizado, la pandemia ha impactado a las economías de forma inesperada y con una severidad sin precedentes en las últimas décadas. Aunque aún es pronto para evaluar las consecuencias socioeconómicas de la actual pandemia a medio y largo plazo, este trabajo constituye un análisis preliminar para analizar los efectos sobre los flujos de remesas de emigrantes y sobre el desarrollo económico de una muestra de países receptores latinoamericanos. Con este fin, examinamos la evolución de una serie de macro-magnitudes que la bibliografía revisada apunta como importantes determinantes de las remesas, tales como el PIB y el empleo en los países que las envían, así como el PIB y el tipo de cambio en los países que las reciben, para de esta forma, relacionarlos con la incidencia de la COVID-19. Además, comparamos la dinámica de las remesas en esta crisis con lo acontecido durante y después de la crisis financiera global de 2008.

Del análisis se desprende que la crisis ha afectado de manera desigual a los países receptores y mientras que, en algunos de ellos, como México la caída interanual de los flujos de remesas en abril de 2020 fue apenas del $3 \%$, en otros, como Bolivia y Paraguay, fue mucho más pronunciada alcanzando más del $60 \%$. Estos dos países reciben remesas principalmente de Argentina y Chile, que han sufrido confinamientos largos y caídas importantes en la actividad económica, lo cual sumado a la apreciación de la moneda local en Bolivia y Paraguay dificulta que las remesas tengan su usual efecto amortiguador ante las crisis. Para el caso de México, es posible que las ayudas estatales recibidas por los trabajadores residiendo en Estados Unidos hayan podido aliviar las caídas en el empleo y posibilitado la continuidad en los envíos. A pesar de todo, la evolución de la variación interanual de los envíos entre abril y junio de 2020 presenta forma de V para los siete países analizados, habiéndose recuperado temporalmente la dinámica de crecimiento de los flujos de remesas tras el primer confinamiento, aunque no los valores absolutos respecto al 2019.

En cuanto a la comparación con la crisis financiera global de 2008, el análisis descriptivo de los datos nos permite concluir que el impacto de la COVID-19, al menos en el corto plazo, es mucho más profundo y diferenciado, pues afecta en mayor medida -vía desempleo-a los migrantes y conlleva, a su vez, a una suspensión de la migración internacional. 
Se podrían anticipar tres factores importantes que afectarán a medio plazo la migración y los flujos de remesas y que están estrechamente relacionados con la pandemia. En primer lugar, la velocidad de recuperación del empleo y el crecimiento económico global después de esta crisis sin precedentes será crucial. En segundo lugar, la gradual transformación hacia una economía digitalizada y robotizada cobrará si cabe mayor importancia con la pandemia como estrategia para reducir costes de transacción y laborales (Chernoff et al., 2020). Finalmente, el aumento de las restricciones a la movilidad internacional tendrá también importantes consecuencias para los envíos de remesas.

En relación con el primer factor, un efecto agravante, no observado en crisis económicas anteriores, es el deterioro de las condiciones de salud y la falta de cobertura sanitaria, que afectan desproporcionalmente a los inmigrantes trabajando en condiciones de informalidad o ilegalidad, condiciones estas que pueden tornarse más frecuentes en el medio plazo tal y como sucedió tras la crisis financiera de 2008. Por este motivo será importante que los Gobiernos amplíen las coberturas sanitarias para mitigar dicho efecto.

En cuanto al segundo factor, la mayor digitalización y robotización, una tendencia ya en curso antes de la pandemia y que se está profundizando con ella, contribuirá a disminuir la demanda laboral de trabajadores no cualificados, y asimismo, las oportunidades de empleo para los migrantes con el consecuente impacto negativo sobre las remesas. Políticas que abran la puerta a algún mecanismo de renta básica o de ingreso mínimo vital que incluyan a los migrantes podrían mitigar el impacto.

El tercer factor incide en el hecho de que las restricciones de movilidad internacional impuestas podrían reducir la migración laboral en los años venideros revirtiendo la tendencia observada en la década pasada que iba acompañada de un aumento de los flujos de remesas. Acuerdos específicos entre países o bloques económicos deberían mediar para frenar o moderar dichas restricciones.

Dada la incertidumbre aparejada a los actuales acontecimientos, es crucial investigar la incidencia precisa de estos factores y para ello, como agenda de investigación futura, será de crucial importancia estimar los efectos a corto plazo de la COVID-19 sobre las remesas y a través de estas sobre la renta y pobreza en los países receptores de las mismas. Ello será más factible cuando los datos del tercer y cuarto trimestres de 2020 estén disponibles para una muestra amplia de países. La metodología que se propone utilizar es la estimación de un modelo econométrico de datos de panel en dos etapas. La primera consiste en la estimación de un modelo de gravedad para estimar los determinantes de los flujos bilaterales de las remesas, incluyendo entre ellos el stock de migrantes, el desarrollo financiero y la renta per cápita en origen y destino, así como también, los costes de la transacción, la adopción de nuevas tecnologías, los periodos de confinamiento y la incidencia de la COVID-19. La segunda etapa consistiría en estimar un modelo de crecimiento económico en el cuál, además de los factores tradicionales, se incorporen las entradas de remesas obtenida a partir de la agregación por país de las predicciones obtenidas en la primera etapa. 
Aunque queda pendiente cuantificar la magnitud de los efectos a medio y largo plazo de la crisis COVID-19 en el sector de envío de remesas, es importante que las instituciones y Gobiernos contribuyan a afianzar el papel amortiguador que las mismas juegan en periodos de crisis. En este sentido, será decisivo el avance en el cumplimiento de los ODS con independencia de lo que acontezca en el entorno macroeconómico. De acuerdo con el ODS 10.c, los países se comprometen a reducir a menos del $3 \%$ los costos de transacción de las remesas de los migrantes y eliminar los corredores con un costo superior al $5 \%$. Siendo Latinoamérica una de las regiones con costos de transacción más elevados, avances en este sentido contribuirían a mitigar los impactos adversos de la caída en las remesas derivados de la pandemia.

\section{Referencias bibliográficas}

Acosta, P., Calderón, C., Fajnzylber, P., \& Lopez, H. (2008). What is the Impact of International Remittances on Poverty and Inequality in Latin America? World Development, 36(1), 89-114.

Adams J. R., \& Richard, H. (2011). Evaluating the Economic Impact of International Remittances on Developing Countries Using Household Surveys: A Literature Review. Journal of Development Studies 47(6), 809-828.

Ahmed, J., Mughal, M., \& Martínez-Zarzoso, I. (2020). Sending Money Home: Transaction Cost and Remittances to Developing Countries. University of Göttingen, Germany.

Ahmed, J., \& Martínez-Zarzoso, I. (2016). Blessing or Curse: The Stabilizing Role of Remittances Compared with other Financial Flows. Journal of South Asian Development, 11(1), 38-66.

Amuedo-Dorantes, C., \& Pozo, S. (2010). Accounting for Remittance and Migration Effects on Children's Schooling. World Development, 38(12), 1747-1759.

Buch, C. M., \& Kuckulenz, A. 2010. Worker Remittances and Capital Flows to Developing Countries. International Migration 48(5), 89-117.

Bertoli, S., \& Marchetta, F. (2014). Migration, remittances and poverty in Ecuador. The Journal of Development Studies 50(8), 1067-1089.

Bucheli, J. R., Bohara, A. K., \& Fontenla, M. (2018). Mixed effects of remittances on child education. IZA Journal of Development and Migration, 8(10), 1-18. https://doi.org/10.1186/s40176-017-0118-y

Caruso, G., Canon, C. G., \& Mueller, V. (2020). Spillover effects of the Venezuelan crisis: migration impacts in Colombia. Oxford Economic Papers.

Catrinescu, N., Leon-Ledesma, M., Piracha, M., \& Quillin, B. (2009). Remittances, institutions, and economic growth. World Development, 37(1), 81-92.

Cazachevici, A., Havranek, T., \& Horvath, R. (2020). Remittances and economic growth: A meta-analysis. World Development, 134, 105021. 
Chami, R., Fullenkamp, C., \& Jahjah, S. (2003). Are Immigrant Remittances Flows a Source of Capital for Development (No. 189). IMF Working Paper.

Chernoff, A. \& Warman, C. (2020). COVID-19 and Implications for Automation (Working Paper No. w27249). National Bureau of Economic Research.

Cruz-Zuniga, M. (2011). On the path to economic growth, do remittances help? Evidence from panel VARs. The Developing Economies, 49(2), 171-202.

Fix, M., Papademetriou, D. G., Batalova, J., Terrazas, A., Lin, S. Y. Y., \& Mittelstadt, M. (2009). Migration and the global recession. Migration Policy Institute, 2.

International Monetary Fund (2019). Fintech in Latin America and the Caribbean: Stocktaking (No. 19/17). IMF Working Paper.

Jadotte, E., \& Ramos-Morilla, X. (2016). The effect of remittances on labour supply in the Republic of Haiti. The Journal of Development Studies 52(12), 1810-1825.

Lim, S. \& Morshed, A.K.M. (2015). International migration, migrant stock, and remittances: Reexamining the motivations to remit. The Quarterly Review of Economics and Finance 57(C), 101-115.

Lueth, E., \& Ruiz-Arranz, M. (2008). Determinants of bilateral remittance flows. The BE Journal of Macroeconomics 8(1), 1-20.

McCracken, S., Ramlogan-Dobson C. \& Stack, M. M. (2017). A gravity model of remittance determinants: evidence from Latin America and the Caribbean. Regional Studies 51(5), 737-749.

Mohapatra, S., Joseph, G. \& Ratha, D. (2012). Remittances and natural disasters: ex-post response and contribution to ex-ante preparedness. Environment, Development and Sustainability 14(3), 365-387. https://doi.org/10.1007/s10668-0119330-8

Organización Internacional para las Migraciones, OIM (2013). Migrantes Paraguayos en Argentina: Población, instituciones y discursos. Cuadernos Migratorios No.4.

Ramírez, M. D., \& Sharma, H. (2009). Remittances and growth in Latin America: A panel unit root and panel cointegration analysis. Estudios Económicos de Desarrollo Internacional 9(1), 1-36.

Rapoport, H. \& Docquier, F. (2006). The Economics of Migrants' Remittances. In S-C. Kolm and J.M. Ythier, (Eds), Handbook of the Economics of Giving, Altruism and Recipocity, Volume 2. Elsevier.

Ratha, D. K., De, S., Kim, E. J., Plaza, S., Seshan, G. K., \& Yameogo, N. D. (2020). COVID-19 crisis through a migration lens. Migration and Development Brief 32. The World Bank.

http://documents.worldbank.org/curated/en/989721587512418006/COVID-19Crisis-Through-a-Migration-Lens

Sirkeci, I., Cohen, J. H. \& Ratha, D. K. (Eds.) (2012). Migration and remittances during the global financial crisis and beyond. The World Bank. 
Sousa, L. D., \& García, A. (2018). Remittances and labor supply in the Northern Triangle. Policy Research Working Paper, vol. 8597. The World Bank. https:// doi.org/10.1596/1813-9450-8597

Vacaflores, D. (2018). Beyond Altruism and Self-interest: The Growing Importance of External Factors in the Determination of Remittances Flowing to Latin America. International Economic Journal 32(2), 235-255. 